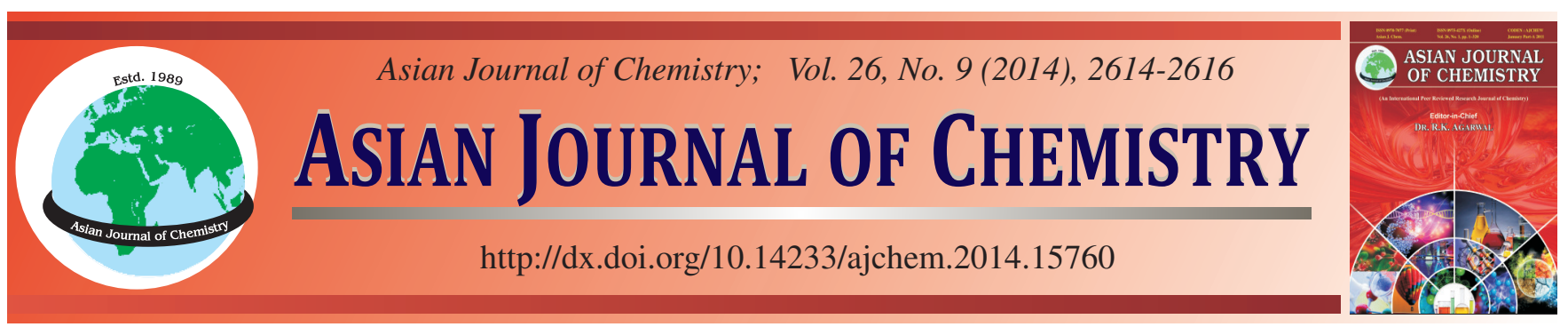

\title{
Formulation and in vitro Evaluation of Gastro Retentive Oral Hydrogel Tablets Containing Cefditoren
}

\author{
G. Venugopala Rao, Ganesh Gadamsetty and N.C. Sarada*
}

School of Advanced Sciences, VIT University, Vellore-632 014, India

*Corresponding author: E-mail: venumpharma@gmail.com

Received: 13 May 2013;

Accepted: 18 September 2013;

Published online: 28 April 2014;

AJC-15080

Cefditoren pivoxil gastro retentive tablets were prepared to achieve the gastro retentive effect in order to improve its absorption and bioavailability. Oral hydrogel tablets were prepared by varying concentrations (CHT1-CHT7) of carbopol and sodium alginate. The precompression parameters and post compression parameters were within acceptable pharmacopeial limits. The swelling index indicates that the formulations prepared with sodium carbonate have good porosity. The in vitro dissolution behaviour for the formulation of (CHT7) containing carbopol $(100 \mathrm{mg})$ and sodium alginate $(20 \mathrm{mg})$ were found to have good controlled release of $91.45 \%$ in the $12 \mathrm{~h}$.

Keywords: Cefditoren pivoxil, Antibiotic, Sodium alginate, Gastro retentive.

\section{INTRODUCTION}

Cefditoren pivoxil $\left(\mathrm{C}_{25} \mathrm{H}_{28} \mathrm{~N}_{6} \mathrm{O}_{7} \mathrm{~S}_{3}\right)$ is a semi-synthetic third generation cephalosporin exhibiting bactericidal action by inhibiting cell wall synthesis. Cefditoren pivoxil is used for the treatment of uncomplicated skin and structure skin infections. Cefditoren pivoxil has a broad spectrum of activity against gram negative and gram positive bacterial infections including strains of Staphylococcus pyrogenes, Haemophilus influenza, Klebsiella pneumonia and Staphylococcus aureus ${ }^{1}$. The molecular weight of cefditoren pivoxil is 620.73 and chemical structure is shown in Fig. 1. Cefditoren pivoxil is a prodrug which get hydrolyzed by esterase during absorption to generate the active drug cefditoren followed by the distribution in blood ${ }^{2}$.

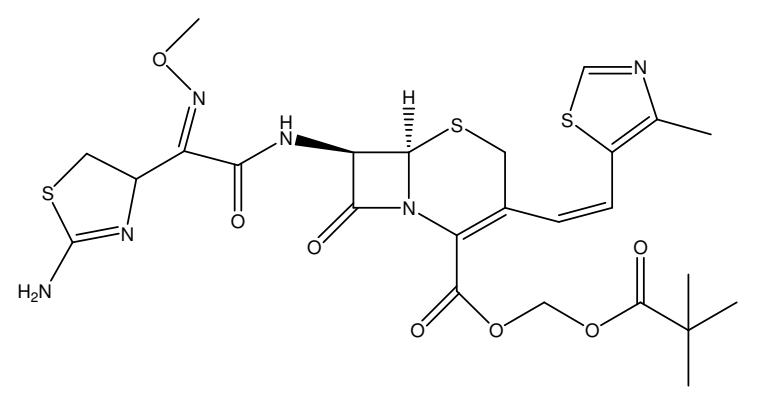

Fig. 1. Chemical structure of cefditoren pivoxil

Novel drug deliveries like gastro retentive dosage forms were the recent advances for delivering the drug molecules to the upper gastro intestinal tract for prolonging the drug release and to improve the absorption ${ }^{3}$. Hydrogel is a three dimensional polymeric network of hydrophilic chains which are cross linked either through physical or chemical bonding. Hydrogel absorbs water to swell in the presence of excess water because of the hydrophilic nature of polymeric chains. The development of super porous hydrogels was formulated with an aim to hold the dosage form in the gastric environment ${ }^{4}$. These drug delivery systems swells up rapidly in the stomach and maintain its uniformity throughout the stomach environment and further helps in releasing the drug. The objective of present study is to formulate and evaluate cefditoren pivoxil gastro retentive tablets based on gel forming polymers using carbopol (940P), sodium alginate and effervescent agent sodium bi carbonate which will help the dosage form to retain in stomach.

\section{EXPERIMENTAL}

Cefditoren pivoxil was a generous gift sample from Hetero drugs (Hyderabad, India), Carbopol 940 and sodium alginate was procured from Pure Chem Laboratory. Magnesiumstearate, Lactose and all other solvents and reagents used were of analytical reagent grade were procured from SDFCL, India.

Preparation of hydrogel tablets: The tablet ingredients were weighed accurately as mentioned in Table- 1 . The above ingredients were then passed through a 20 mesh sieve and properly mixed. Finally the blends were mixed for $5 \mathrm{~min}$ after the addition of magnesium stearate and talc. The blends were compressed using a 16 station rotary punch tablet machine (Cadmach, Germany) having caplet shaped concave punches ${ }^{5}$. 


\begin{tabular}{|c|c|c|c|c|c|c|c|}
\hline \multicolumn{8}{|c|}{$\begin{array}{c}\text { TABLE-1 } \\
\text { COMPOSITION OF HYDROGEL TABLETS }\end{array}$} \\
\hline Ingredients (mg/tablet) & CHT1 & CHT2 & CHT3 & CHT4 & CHT5 & CHT6 & CHT7 \\
\hline Drug & 200 & 200 & 200 & 200 & 200 & 200 & 200 \\
\hline Carbopol & 50 & 75 & 100 & 100 & 100 & 100 & 100 \\
\hline $\mathrm{NaHCO}_{3}$ & 30 & 30 & 30 & 30 & 30 & 30 & 30 \\
\hline Lactose & 212 & 187 & 162 & 157 & 152 & 147 & 142 \\
\hline Sodium alginate & - & - & - & 5 & 10 & 15 & 20 \\
\hline Magnesium stearate & 5 & 5 & 5 & 5 & 5 & 5 & 5 \\
\hline Talc & 3 & 3 & 3 & 3 & 3 & 3 & 3 \\
\hline
\end{tabular}

Post compression parameters of hydrogel tablets: Hydrogel tablets were evaluated for drug content uniformity, weight variation, friability, thickness and hardness according to the specifications of British pharmacopoeia. Drug content was analyzed using Shimadzu UV-visible spectrophotometer (1700) at $271 \mathrm{~nm}$ and the $\%$ of the drug content was estimated ${ }^{6}$.

in vitro Dissolution test: The study was carried out in $900 \mathrm{~mL}$ of $0.1 \mathrm{~N} \mathrm{HCl}$ at $75 \mathrm{rpm}$, which was maintained at $37 \pm$ $0.5^{\circ} \mathrm{C}$ using the USP apparatus type II (Electrolab Disso 8000). $5 \mathrm{~mL}$ of samples were withdrawn at regular intervals of $1 \mathrm{~h}$ and the absorbance was measured at $271 \mathrm{~nm}$ using a Shimadzu UV spectrophotometer 1700. The sink condition was maintained by replacing with fresh buffer medium. The dissolution study was carried out for $24 \mathrm{~h}$ followed by mathematical treatment of the solved dissolution data ${ }^{7}$.

\section{RESULTS AND DISCUSSION}

Evaluation of hydrogel tablets: The formulated hydrogel tablets were evaluated for post compression parameters such as hardness, content uniformity, thickness, friability and weight variation tests (Table-2). Table-2 reveals that the content uniformity, friability and weight variation tests were within the limits as per the pharmacopeial specifications. Table-2 also reveals that the thickness and hardness increases as the concentration increases which helps to release the drug in a controlled release manner. Fig. 2 showed that the in vitro drug release changes according to the polymer concentration. The drug release gets retarded as the carbopol concentration increases (CHT1-CHT3), but the release cannot be controlled up to the desired extent. As carbopol is a cross linked acrylic polymer which has the potential to extend the release of drugs from gastro retentive delivery systems, they are an efficient hydrophilic matrix forming excipient and they enable the uniform dispersion of drugs in a polymeric matrix using direct compression. When such tablets come in to contact with dissolution media, the external surface of the tablet becomes hydrated, swells and forms a gel like layer that further controls the release of the drug from the tablets. Further, in order to

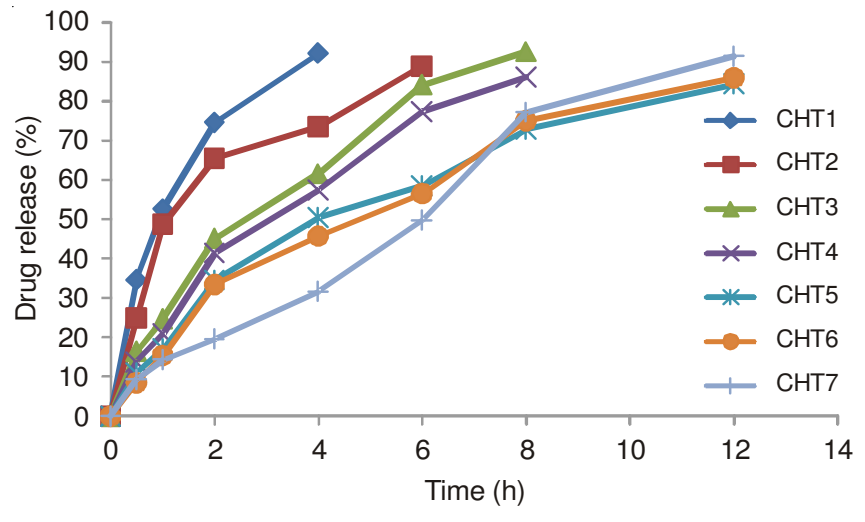

Fig. 2. In vitro drug release of prepared hydrogels

extend the release, sodium alginate was used (CHT4-CHT7) along with carbopol. From the above results, Fig. 2 clearly finds that the formulation CHT7 showed better controlled release behaviour when compared to all other formulations $(91.45 \%)$. Thus the formulation CHT7 has been optimized and used for further studies.

Swelling index: Swelling index ${ }^{8}$ was carried out for $12 \mathrm{~h}$. Fig. 3 showed that $75 \%$ of swelling index was observed for the formulation CHT7 due to the higher concentration of sodium alginate. No destruction of the tablet is seen even though there is a faster swelling. This might be due to the presence of carbopol and the effervescent agent sodium bicarbonate. This further confirms that the prepared tablets have the capability to withstand in the gastro-intestinal tract as well as in the gastro-intestinal environment.

\section{Conclusion}

The above research work has been carried out with the aim of controlling the release of cefditoren pivoxil with sodium carbonate, carbopol and sodium alginate. With the use of above mentioned excipients in different concentrations the gastro retentive effect was successful. The tablets were formulated by direct compression. All the physical parameters were in acceptable range as per the pharmacopeial specifications. The

\begin{tabular}{cccccc}
\multicolumn{7}{c}{ TABLE-2 } \\
POST COMPRESSION PARAMETERS OF HYDROGEL TABLETS \\
\hline Formulations & Weight variation $(\mathrm{mg})$ & Drug content $(\%)$ & Hardness $\left(\mathrm{kg} / \mathrm{cm}^{2}\right)$ & Thickness $(\mathrm{mm})$ & Friability $(\%)$ \\
\hline CHT1 & $500 \pm 1.048$ & $95.37 \pm 0.77$ & $9.33 \pm 0.67$ & $4.19 \pm 0.025$ & 0.18 \\
CHT2 & $501 \pm 1.36$ & $94.04 \pm 0.91$ & $8.66 \pm 0.29$ & $4.19 \pm 0.010$ & 0.25 \\
CHT3 & $499 \pm 0.92$ & $95.03 \pm 0.16$ & $9.00 \pm 0.37$ & $4.19 \pm 0.061$ & 0.39 \\
CHT4 & $500 \pm 1.80$ & $96.88 \pm 0.29$ & $9.30 \pm 0.42$ & $4.19 \pm 0.042$ & 0.06 \\
CHT5 & $501 \pm 1.07$ & $95.32 \pm 0.66$ & $8.66 \pm 0.40$ & $4.19 \pm 0.035$ & 0.19 \\
CHT6 & $502 \pm 1.73$ & $95.34 \pm 0.90$ & $9.33 \pm 0.57$ & $4.22 \pm 0.039$ & 0.12 \\
CHT7 & $501 \pm 1.61$ & $96.26 \pm 1.00$ & $9.33 \pm 0.70$ & $4.20 \pm 0.040$ & 0.17 \\
\hline
\end{tabular}




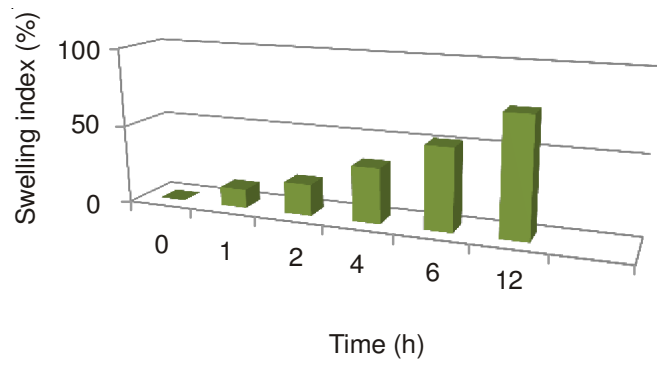

Fig. 3. Swelling behaviour of CHT7 for $12 \mathrm{~h}$

formulation CHT7 (20\% carbopol, $6 \%$ sodium carbonate and $4 \%$ of sodium-alginate) showed a controlled release with gastro retentive effect when compared to other formulations, which was further confirmed by the swelling index. Further to improve in vitro drug release till $24 \mathrm{~h}$, additional optimization of formulation is needed.

\section{REFERENCES}

1. E.A. Balbisi, Pharmacotherapy, 22, 1278 (2002).

2. Spectracef, Physicians' Desk Reference, Montvale (NJ), Thompson PDR, 7, 2824 (2005).

3. K.C. Waterman, Pharm. Dev. Technol., 12, 1 (2007)

4. J. Chen, W.E. Blevins, H. Park and K. Park, J. Control. Rel., 64, 39 (2000).

5. R. Bushra, M.H. Shoaib, N. Aslam, D. Hashmat and M.U. Rehman, Pak. J. Pharm. Sci., 21, 113 (2008).

6. L.V. Allen, N.G. Popvich and H.C. Ansel, in eds.: Lippincott Williams and Wilkins, Pharmaceutical Dosage Forms and Drug Delivery System, Wolters Kluwer Company, Philadelphia, Baltimore, edn. 8, p. 227 (2005).

7. The United State Pharmacopoeia, (USP 24 NF 19), Convention INC., 1462, 1913 (2000).

8. G. DiColo, A. Baggiani, Y. Zambito, G. Mollica, M. Geppi and M.F. Serafini, Int. J. Pharm., 310, 154 (2006). 